\title{
Molecular Microbial Diagnostic in Lung Transplant Recipients
}

\author{
Sebastián Méndez-Álvarez ${ }^{1,2^{*}}$, Gerardo Pulido-Reyes ${ }^{1}$, Javier Donate-Correa ${ }^{1}$, \\ Arturo Soriano Benítez de Lugo ${ }^{3}$ \\ ${ }^{1}$ Research Unit, Hospital Universitario Ntra. Sra. de Candelaria, Santa Cruz de Tenerife, Spain; ${ }^{2}$ Microbiology Department, \\ Universidad de La Laguna, Tenerife, Spain; ${ }^{3}$ General Surgery Service, Hospital Universitario Ntra. Sra. de Candelaria, Santa Cruz de \\ Tenerife, Spain. \\ Email: *sebastianmendez@funcis.org
}

Received July $16^{\text {th }}, 2012$; revised August $20^{\text {th }}, 2012$; accepted August $31^{\text {st }}, 2012$

\begin{abstract}
The appearing of microbial lineages carrying multiple dangerous loci are results from the extensive use of antibiotics and has brought a huge increase in the infections-derived morbidity and mortality, which is critical in the hospital setting. Etiologic agents of these non easily tractable infections include bacteria, such as the genus Staphylococcus, but also fungi and virus. Alterations in the immune system allow these organisms to invade and affect the functionality of any tissue, organ or system of the human being. Pulmonary infection occurs as result of deficient lung systemic defence mechanisms which could be altered by medical treatments or by environmental factors. The infective agents commonly gain access to the lung by air, but also by blood or lymphatic system. Staphylococcus aureus strains that share antibiotic resistance and virulence factors represent the aetiological agent responsible of many cases of bacterial pneumonia, thoracic surgery postoperative infections, and diverse tissue infections, resulting in significant disease and morbidity in recipient patients after lung transplantation. The control and treatment of Staphylococcus infections, especially methicillin resistant strains, need for developing reliable and rapid methods of detection and characterization of these microorganisms. Nowadays, new insights into the diagnostic and epidemiology of MRSA and other pathogenic staphylococci have been developed employing molecular methods. This has meant an important advance in the diagnostic and treatment plans of such infective bacteria.
\end{abstract}

Keywords: Molecular Microbial Diagnostic; Lung Transplant Recipients

\section{Introduction}

The progressive increment of microbial infections caused by highly virulent multiple resistant strains has become one of the most worrying health evils. Selective pressure resulting from the extensive use of antibiotics during last 35 years has brought to the emergence, selection and dissemination of resistance and virulence genes among pathogenic microorganisms. All these factors have brought us the risk of suffering non tractable infections. Clear examples are the growing appearance and spreading of resistance in Gram-positive bacteria such as the genus Staphylococcus or Mycobacterium; Gram-negative bacteria as Pseudomonas; fungi as Aspergillus; virus as $C y$ tomegalovirus (CMV) and several parasites [1-7]. The constant and fast worldwide dispersion of microbial lineages carrying multiple dangerous loci has caused a huge increase in the infections-derived morbidity and mortality. These aspects were already observed by WHO

\footnotetext{
"Corresponding author.
}

in 2001 in the document "Infection control programs to control antimicrobial resistance" [8].

Infections caused by non easy tractable microbes are occurring globally and can affect any tissue, organ or system of the human being depending on the etiologic agent. Pulmonary infection occurs as result of the interference of immunosuppressive factors with the normal lung systemic defence mechanisms [9]. Several factors interfere with normal lung defence facilitating lung infection: depressed bactericidal and phagocyte function of macrophage caused by starvation, alcohol ingestion, hypoxia, uraemia, air pollutant, cigarette smoke and previous viral infection; drop off or loss of cough reflex leading to aspiration caused by drug, anaesthesias or coma; pulmonary edema; collection of secretion; poor mucociliary elevator function from smoking, infection, etc.; cystic fibrosis, airway obstruction, chronic bronchitis, and others [10-12].

In turn, pathogenic organisms gain access to the lung by different routes: through the airways; through the 
bloodstream; by traumatic implantation; or by a direct spread across the diaphragm through the lymphatic system. The most common route is the airways. Airway spread can result from inhalation of the organism as an aerosol on droplet nuclei. This is a major mechanism of spread of many viral infections and of tuberculosis $[3,13$, 14]. Development of infection often begins with colonization of the upper respiratory tract by potential pathogens followed by aspiration into the lower respiretory tract. A high diversity of microbes can be responseble of infection and associated decline of the respiratory system. Among all of them, our group has mainly specialized in the study of the Gram-positive bacterial genus Staphylococcus, principally $S$. aureus $[15,16]$. This microorganism is the aetiological agent responsible of many cases of bacterial pneumonia, thoracic surgery postoperative infections, and diverse tissue infections, resulting in significant disease and morbidity in recipient patients after lung transplantation.

\section{Thoracic Surgery}

Thoracic surgery is the field of medicine involved in the surgical treatment of diseases affecting organs inside the thorax (the chest). Generally, it involves treatment of conditions of the lungs, chest wall, and/or diaphragm.

A Thoracic Surgeon provides the operative, per operative care and critical care of patients with acquired or congenital pathologic conditions within the chest. The thoracic surgeon function includes pathologic conditions of the lung, oesophagus and chest wall, abnormalities of the great vessels, tumours of the mediastinum, and diseases of the diaphragm and pericardium. Management of the airway and injuries of the chest are within the scope of the specialty [11,17-19].

Major complications following thoracic surgery fall into two categories: respiratory difficulties and wound infections. These postoperative infections (PIs) are serious complications of thoracic surgery and S. aureus infective agent has been described as one of the most important pathogens associated with surgical wound infections. Specifically methicillin-resistant S. aureus (MRSA) continues to pose a major threat to the lung and cardiovascular surgery patients [20].

\section{Lung Transplantation}

Lung transplantation is an important therapeutic treatment for many patients with life-threatening pulmonary diseases; however, long-term survival is still relatively limited compared with other solid organ transplants [21]. Lung transplantation has many potential posttransplant complications with PIs being a major contributor. Compared with recipients of other solid organ transplants (SOT) lung transplant recipients (LTR) are at particularly high risk of infectious complications due to a variety of factors associated to the higher state of immunosuppression, the direct and continuous contact with pathogens, bacterial contamination from the donor lung, the denervation of the allograft resulting in diminished cough reflex and mucociliary function, the impaired lymphatic drainage due to its disruption and the risk of cross-contamination of the transplanted lung by the native lung in single LTR [22,23]. Infectious complications substantially contribute to low-grade outcomes after lung transplantation (LTx) compared to other types of SOT, being the average 1-year survival rate approximately $85 \%$, with a 5-year survival rate of 53\% [17,18,22-25].

During the last years, several articles concerning infections in LTR have been published. In particular, important information has been published regarding CMV prophylaxis and treatment, and fungal infections following lung transplantation [11,14,23-27]. Furthermore, recent studies have shown that the incidence of MRSA has dramatically increased in lung transplant programs $[18,28]$. These studies highlight the importance of implementing preventive measures for MRSA colonization before, during and after lung transplantation.

\section{Bacterial Infections}

Pneumonia and influenza account for a high number of deaths all over the world and are the fifth leading cause of death, exceeded only by heart disease, cancer, cerebrovascular disease and accidents. Pneumonia can be broadly defined as any infection in the lung [29]. Acute pneumonias may be caused by pyogenic bacteria that primarily induce neutrophilic exudates in alveoli, bronchioles and bronchi or by a miscellaneous group of microorganisms that induce predominantly peribronchiolar and interstitial mononuclear inflammation $[9,22,23,25,29]$.

Bacterial infections occur in two frequently overlapping morphologic patterns: bronchopneumonia and lobar pneumonia. They both can be caused by a variety of Gram-positive and Gram-negative organisms [4,30]. Depending on bacterial virulence and host resistance, the same organism may in one case cause bronchopneumonia and in another case lobar pneumonia and sometimes intermediate involvement.

In the first 3 months after transplantation, bacterial infections are responsible for most deaths. Pneumonia is the most common type of infection in LTR with Gramnegative bacteria (GNB) being responsible for the bulk of disease, most commonly with Pseudomonas aeruginosa and other GNB such as Burkholderia spp (formerly known as Pseudomonas cepacia) $[23,30]$. Cystic fibrosis (CF) patients infected with B. cepacia have historically been prone to postoperative infectious complications and poor outcomes. Because of this situation, many transplant centres consider infection with Burkholderia spe- 
cies a contraindication to lung trans- plant in CF [10,17]. However, recent publications have signalled that postoperative infections in CF after lung transplantation can caused by a variety of microorganisms: Pseudomonas aeruginosa, Mycobacterium spp, Aspergillus spp, and Staphylococcus spp [4,6,7,17,24,30].

Certain Burkholderia species, potentially including $B$. gladioli and some $B$. cenocepacia, cause a greater risk for death than do other Burkholderia species. Although Burkholderia infection by itself should not be considered an absolute contraindication for LTx, selection for transplant should consider the specific Burkholderia species present (9 genetically distinct species or genomovars are typified) [22], since there is an increased risk of death among patients infected with $B$. cenocepacia, versus those infected with non-cenocepacia species [23].

Bronchiolitis obliterans syndrome (BOS) is a major long-term complication following LTx, affecting approximately one-third of recipients by 3 years [31]. Colonization with GNB, particularly Pseudomonas, may play a role in the aetiology of BOS, so strategies aimed at reducing colonization may decrease rates of BOS and improve long-term outcomes [22]. Chronic pneumonia is caused by bacteria as Nocardia or Actinomyces species and some parasites, fungi and intracellular bacteria $[2,13,25]$.

Nosocomial pneumonias develop in a number of hospitalized patients especially in critically ill patients admitted to intensive care units (ICUs). With the use of potent therapies that deliberately or incidentally produce immunosuppression there is a great risk of developing respiratory infection. The Gram-positive bacteria (GPB) Streptococcus pneumoniae is often colonizing the throat, and is the most frequent agent causing bacterial pneumonia. Second malevolence GPB causing pneumonia are Staphylococcus spp, especially $S$. aureus mainly if it harbours methicillin or other antibiotic resistance and/or virulent factors as Panton-Valentine Leukocydin (PVL), cell interfering clumping factors, etc. [27,32,33]. Other bacteria also involved in causing infectious in the LTR can be Chlamydia pneumoniae, and Clostridium difficile. Moreover, recently have been reported that nontuberculous mycobacterial (NTM) may be an underrecognized cause of posttransplant complications and certain candidates for LTx should be screened for NTM infections $[3,17]$.

Fungal infections are also a frequent complication in LTR and among SOT recipients [51]. LTR appears to be the highest with an incidence between $15 \%$ and $35 \%$, with an overall mortality of $80 \%[25,34]$. Aspergillus and Candida species cause the majority of fungal infections in lung transplant recipients; Cryptococcus spp, the agents of mucormycosis, endemic fungi (Histoplasma,
Coccidioides, and Blastomyces spp), Scedosporium spp, Fusarium spp, and dermatiaceous molds are other important causes [7,29]. The most common manifestations of aspergillosis in LTR are tracheobronchitis (37\%), invasive pulmonary aspergillosis $(32 \%)$, bronchial anastomosis infections $(20 \%)$ and disseminated disease $(10 \%)$ $[6,7]$. The incidence and timing of invasive aspergillosis (IA) in LTR has changed over time. Earlier publications had reported the incidence to be higher during the first year after transplantation but recent data suggest a much lower incidence during the first year and a more delayed presentation [11,25].

Some characteristics of Fungal Infections are unique in LTR [25]. Tracheobronchial aspergillosis occurs only in lung transplant recipients, typically within three months of transplantation [6]. Predisposing factors include preoperative colonization with Aspergillus, early airway ischemia, stenotic airways, the presence of an airway stunt, single lung transplantation, hypogammglobulinemia, CMV infection, use of alemtuzumab or thymoglobulin induction therapies and acute rejection requiring augmentation of immunosuppression [6,23]. The diagnosis of IA is by biopsy with culture, accompanied by compatible clinical and radiographic abnormalities. Detection of galactomannan, a polysaccharide cell wall component that is released by Aspergillus organisms during fungal growth, by sandwich enzyme immunoassay in the bronchoalveolar lavage (BAL) appears as promising for its diagnosis [23]. Candidemia usually occurs during the first month following LTx as a result of intensive care unit exposure and recent surgery but in adults no cases of invasive pulmonary candidiasis have been reported following LTx $[11,25]$.

Viruses' infections in lung transplant recipients are preferentially represented by CMV viriasis [24]. LT recipients have the highest risk of developing CMV disease among all SOT recipients, although the risk of CMV has decreased over time [14]. CMV infection contributes to a significant proportion of post-LTx morbidity and mortality but since the introduction of more effective CMV prophylaxis, the burden of CMV infection and disease in recipients of SOT has diminished significantly as well as all-cause mortality in SOT recipients [21]. CMV infection requires evidence of $\mathrm{CMV}$ viral replication trough laboratory testing, whereas CMV disease requires not only CMV viral replication but any evidence of typical symptoms or tissue-invasive disease. CMV infection or disease is initially diagnosed based on the detection of viremia, lately quantitative nucleic acid testing, via polymerase chain reaction (PCR), which has become the most widely accepted method of CMV viral load monitoring [26,35]. Most authorities favours the use of universal CMV prophylaxis (administration of antivirals to 
all at-risk individuals, positive donor or recipient serologic status), including lung transplant recipients, being oral valganciclovir either alone or after a short course of intravenous ganciclovir the usual accepted strategy [5]. A recent trial comparing short-course (3 months) with longcourse (12 months) prophylaxis with valganciclovir in at-risk LTx showed that only $4 \%$ of long-course prophylaxis patients developed CMV disease versus $32 \%$ of short-course patients $[5,17]$.

\section{Staphylococcus aureus Pulmonary Infections. Molecular Diagnostics}

Currently, one of the most worrisome examples about infections caused by pathogenic bacteria is the rising emergence and spreading of antibiotic resistance and virulence factors in Staphylococcus aureus and other members of the genus Staphylococcus [36]. Several population groups are under high risk of suffering infections by pathogenic staphylococci. Staphylococcus spp infections are usual in immunocompromised patients: e.g. postsurgery patients, transplanted patients, persons affected of diseases such as flu, bronchopulmonar pathologies. The control and treatment of Staphylococcus infections is an extremely relevant subject from its health and clinical implications [15]. Moreover, already in the XXI century, the rising dissemination of methicillin resistant strains, antibiotic that was considered the basic agent against these infections, has extremely complicated their erradication. Thereby, there is a growing frequency of appearance of MRSA strains worlwide, especially in hospital settings, although emergence of community acquired infections is also rising up. Furthermore, the frequency of resistance in MRSA against the so called last weapons, as e.g. vancomycin, mupirocin, linezolid, daptomycin, is also increasing. In this context, we have carried out the selection, design and assay of new natural or synthetic molecules with antibiotic activity against staphylococci (patent pending Ref. ES-2498.1, Casero C., Estévez-Braun A., Gutiérrez-Ravelo A., Demo M., Méndez-Álvarez S., Machín-Concepción F.).

The need for developing reliable and rapid methods of detection and characterization of these pathogenic microorganisms has led to new insights into the diagnostic and epidemiology of MRSA and other pathogenic staphylococci. Our working group has been focused on the development of molecular protocols for a sensitive, specific and fast identification of $S$. aureus from clinical samples simultaneously detecting resistance and/or virulence genes [37,38]. The succeed in this goal has meant an important advance in the diagnostic and treatment plans of such infective bacteria. The protocols developed allowed to elucidate the existing association between the presence of the Panton-Valentine leukocidin-encoding gene and a lower rate of survival among hospitalized pulmonary patients with staphylococcal infection [12]. Moreover, combination of different molecular typing methods with classical microbiology ones has permitted us to build up solid MRSA epidemiology studies at both global level and local stage [33,39-44]. These studies constitute a corner stone for the establishment of prevention and treatment measures and can be divided according to the utilization of DNA amplification $[45,46]$.

\subsection{Methods without DNA Amplification}

\subsubsection{Restriction Fragment Length Polymorphisms (RFLPs)}

Since the chromosome is the most fundamental component of identity of the cell, methods measuring this molecule represents a preferred approximation for assessing strain interrelatedness. Restriction patterns originated after chromosomal DNA enzymatic digestion generates a restriction fragment length polymorphism (RFLP), after pulsed field gel electrophoresis (PFGE), which efficiently and accurately allows the differentiation of strains and compare following conventional agarose gel electrophoresis [47].

PFGE methods have been used to evaluate the spread of various antimicrobial resistant bacteria [48]. The finding of isolates that have identical or related restriction endonuclease patterns suggests spread from single strains. Guidelines proposed by Tenover et al. (1997) are often used to for the interpretation of PFGE [45]. With these guidelines, a banding pattern difference of three fragments could have occurred due to a single genetic event and thus these isolates are classified as highly related; differences of four to six restriction fragments are likely due to two genetic events, and differences of greater than seven restriction fragments are due to three or more genetic events. Isolates that differ by three fragments in PFGE analysis may represent epidemiologically related subtypes of the same strain. Conversely, isolates differing in the positions of more than three restriction fragments may represent a more tenuous epidemiologic relation. A number of studies using PFGE and other typing methods indicate that single genetic events, such as those that may alter or create a new restriction endonuclease site or DNA insertions/deletions associated with plasmids, bacteriophages, or insertion sequences, can occur unpredictably even within the time span of a well-defined outbreak (One to three months) [49].

In general, strains are considered identical if they show $100 \%$ similarity and are considered clonally related if they show greater than $80 \%$ similarity (generally comparable to a three-fragment difference as noted above). The typical phylogenic output is the dendrogram, which provides a visual representation of strain lineages, plus genetic similarities and differences between groups.The accuracy and typing MRSA isolates [48]. 


\subsubsection{Plasmid Profiling and Restriction Endonuclease Analysis of Plasmid (REAP)}

Plasmids are DNA molecules that are separate from, and can replicate independently of, the chromosomal DNA. Plasmid sizes vary enormously and can be considered part of the mobilome because they are often associated with conjugation, a mechanism of horizontal gene transfer which typically provides a selective advantage under a given environmental state since may carry genes that provide resistance to antibiotics or the ability to produce proteins that act as toxins [40].

Plasmids are present as variable components of many staphylococcal genomes, and are classified into 4 classes and 15 incompatibility groups which are often responseble for antibiotic resistance. This fact is exploited for typing of multi-resistant staphylococci by separating in agarose gel electrophoresis the isolated intact plasmidic DNA [34]. Thus, the number and size of plasmids is determined in a plasmid profiling. Since extrachromosomal DNA content shows considerable variation, plasmid profiling is suitable for the study of relatively recent epidemiological relationships, whereas analysis of chromosomal DNA reflects more reliably relationships over a longer period of time.

In restriction endonuclease analysis of plasmid (REAP) the isolated plasmid DNA of $S$. aureus is digested separately with the restriction enzymes HindIII and EcoRI. The DNA digests obtained are separated by electrophoresis in agarose gels generating patterns of restriction fragments which allow to determine composite strain types. This method can be complemented with specific gene detection using probes targeting for instance, genes encoding antibiotic resistance [36]. Owing to the possible instability of plasmid, the method shows only moderate reproducibility and therefore an additional typing method is to be used for analysis $[37,40]$.

\subsection{Methods with DNA Amplification}

\subsubsection{Gene-Specific PCR}

Amplification and sequencing conserved genes of bacterial genomic DNA are used for identification of staphylococcal species and their genotypes. In S. aureus the genes encoding 16S rRNA, factor A essential for methicillin resistance (femA), and staphylococcal thermonuclease (nис) (among others) are frequently used for identification at the species level [43].

\subsubsection{Multiplex PCR Assays (MPCR)}

The multiplex PCR (MPCR) assays allows simultaneous amplification of several genes in one reaction mixture. As explained above, MRSA strains constitute a major health care problem; therefore, the availability of sensitive and specific methods for the accurate detection of antibiotic resistance in these bacteria has become an im- portant tool in clinical diagnosis. Since phenotypic typing methods are not discriminating enough and are highly dependent on growth conditions, it is essential to use molecular techniques to stop the spread of multiple-antibiotic-resistant $S$. aureus. These techniques allow a rapid, accurate identification of staphylococci and their resistance type. Thus, fast, sensitive, and specific molecular methods will be an essential diagnostic tool for microbiology laboratories. The use of PCR for the sensitive and specific detection of microorganisms and antibiotic resistance genes is increasing in clinical microbialogy laboratories. There are several reports in the literature describing the use of MPCR for detection of MRSA strains, but most of these protocols are designed to detect only one or two gene fragments from overnight liquid cultures [37].

In 2001, our group described a multiplex PCR assay for the detecting clinically relevant antibiotic resistance genes harbored by some $S$. aureus isolates. Conditions were optimized for the simultaneous detection of regions of the mecA (encoding high-level methicillin resistance), ileS-2 (encoding high-level mupirocin resistance), and femB (encoding a factor essential for methicillin resistance) genes, respectively, from a single colony in a single reaction tube $[35,37,42]$.

These MPCRs offers a rapid, simple, feasible, specific, sensitive, and accurate identification of mupirocin-resistant MRSA clinical isolates and is suitable for diagnostic test in clinical microbiology laboratories, facilitating the design and use of antibiotic therapy $[38,50]$.

\subsubsection{Multiple-Locus Variable Number Tandem Repeat Analysis (MLVA)}

A relatively new method for typing $S$. aureus strains is the multiple-locus variable-number tandem-repeat analysis (MLVA). This technique consists of simultaneous amplification of variable-number tandem repeats of different genes. Several works have tried to determine if MLVA provides enough information to be performed routinely instead of PFGE or MLST, which are more laborious, in the clinical setting. In our laboratory, MLVA was performed as previously described [51], but slightly modified to obtain optimal results and to accelerate the process $[33,52,53]$ concluding that the utilization of MLVA allows to distinguish among different MRSA reservoirs and other circulating MRSA strains. The proven simplicity, low cost, and speed of MLVA enable the performance of routine checkups in patients, mainly via admission screening on surgical wards and in intensive care units, hampering the spread of these strains and therefore reducing the morbidity, mortality, and costs $[28,44,54,55]$.

\subsubsection{Spa Typing of MRSA}

DNA sequence analysis of the protein A gene variable 
repeat region (spa) typing provides a rapid and accurate method to discriminate $S$. aureus outbreak isolates from those deemed epidemiologically unrelated. This technique involves DNA sequencing of short sequence repeats in the polymorphic $\mathrm{X}$ region of the protein A gene of $S$. aureus. The region consists of a variable number of these repeat units which are usually $24 \mathrm{bp}$ in length (Laboratory of HealthCare Associated Infection). Each new base composition of a repeat is assigned an alphanumerical code (r01, r02, etc.) and the repeat succession determines the spa type (e.g. t001, t002, etc.). Spa typing is used throughout Europe and world-wide for reliable, accurate and discriminatory typing of $S$. aureus [both methicillin sensible $S$. aureus (MSSA) \& MRSA]. Over 7000 spa types have been described to date; spa non-typable strains are rare (less than $0.1 \%$ ). There is a standard international nomenclature which is web-enabled and the data are directly comparable between centers and countries. For some S. aureus lineages, the technique has a discriminatory index approaching that of PFGE [56].

\subsection{Multi-Locus Sequence Typing (MLST)}

Multi-locus sequence typing is the molecular method with highest discriminatory capability and is based in characterizing the sequences of $450 \mathrm{bp}$ internal fragments of 7 housekeeping genes: carbamate kinase (arcC), shikimate 5-dehydrogenase (aroE), glycerol kinase (glpF), guanylate kinase (gmk), phosphate acetyltransferase (pta), triose-phosphate isomerase (tpi) and acetyl-CoA $C$-acetyltransferase (yqiL). The sequences obtained are assigned to allele numbers after comparison with a DNA sequences database (www.mlst.net). The allele numbers at each of the seven loci define the allelic profile or sequence type (ST). Novel alleles and STs not found on the MLST website are confirmed by repeating both the PCR and sequencing [49,57].

MLST has been employed for identifying the MRSA and MSSA clones among isolates from patients with serious community- and hospital-acquired infections. This method is suitable for studying both the evolution of MRSA pandemic clones and local epidemiology, and has similar discriminatory power to PFGE [58]. MLST provides generally highly reproducible and comparable results. However, it has the disadvantage of being expensive and technically demanding. Therefore, there are attempts to detect sequence polymorphisms of the 7 loci by other method instead of DNA sequencing (like DNA microarrays).

\subsection{Staphylococcal Cassette Chromosome Mec Typing (SCCmec)}

A remarkable phenomenum that occurs is that MSSA strains become MRSA strains by the acquisition of a staphylococcal cassette chromosome mec (SCCmec) element carrying the mecA gene, which is responsible for methicillin resistance. SCCmec elements are unique genomic islands that are found in staphylococci. These elements have two essential components, the $\mathrm{ccr}$ gene complex (ccr) and the mec gene complex (mec) [59]. The ccr gene complex is composed of $c c r$ genes and surrounding open reading frames (ORFs), and the mec gene complex is composed of the mecA gene, regulatory genes, and insertion sequences upstream or downstream of mecA. Several mec and $c c r$ allotypes have been found among SCCmec elements, what has led to the specific classification. Oliveira and Lencastre (2002) developed a novel method to identify the structural types of SCCmec in MRSA strains based on a MPCR aproximation [59]. To date, several structural-differences in SCCmec elements have been identified. SCCmec typing classifies SCCmec elements in the basis of their structural-differences and is used in epidemiological studies to discriminate MRSA strains or to define a MRSA clone in combination with the genotype of MSSA strain in which a SCCmec element has integrated [53].

The different techniques available for typing MRSA serves to emphasize that none has yet been recognized as the definitive method [54]. In many cases it is necessary use a battery of them, so our group have followed this work line. In 2003, we communicated that we had detected the UK EMRSA-16 multiresistant clone as an epidemic clone in a university hospital in Tenerife, Spain (Hospital Universitario Ntra. Sra. de Candelaria, HUNSC) [43]. To our knowledge, this was the first time that hospital establishment of the EMRSA-16 clone in Spain had been reported. We have ensured the clonal nature of our isolates by development and integrative analyses of different molecular approaches: PFGE, SCCmec multiplex PCR assay, MLST, MLVA and spa typing. Results from these analyses showed that the clone was ST36-MRSA-II. Subsequently, we tracked MRSA clones during a 5-year period in the HUNSC [36].

As explained above, MRSA is the most common cause of serious hospital-acquired infections. Infections of the respiratory tract by $S$. aureus can be more severe if the infecting strain produces the Panton-Valentine leukocidin (PVL) [32]. The serious impact of PVL-positive $S$. aureus infections seems to be associated with pulmonary complications. In 2007, we hypothesized that PVL-positive MRSA is associated with mortality in patients with $S$. aureus pneumonia [12]. The presence of PVL differed significantly between dead and living patients, since all of the PVL-positive patients died. The findings of this study may have some implications for clinical decision making. PVL-positive MRSA strains seem to be dangerous for pulmonary patients, so we recommend screening 
for the presence of PVL when an MRSA is detected in such patients. The presence of PVL could be detected by simple PCR amplification and sequencing specific genes.

In other study [52], MLVA was used to perform 292 MRSA isolates previously characterized by PFGE, MLST, and SCCmec typing. This study demonstrated the ability of MLVA to distinguish among different MRSA reservoirs and other circulating MRSA strains in the HUNSC. The proven simplicity, low cost, and speed of MLVA enable the performance of routine checkups in patients, mainly via admission screening on surgical wards and in intensive care units, hampering the spread of these strains and therefore reducing the morbidity, mortality, and costs.

In a recent published article [53], a wide variety of MRSA clones was revealed, including an emergent ST and two new spa types. The PVL genes were found in isolates belonging to unrelated lineages, what could indicate different independent introductions of PVL-positive strains in Tenerife. Moreover, we detected that hospital MRSA clones had spread to the community and are now circulating in both environments [44, 53].

Definitely, laboratory screening for MRSA is a complex balance between speed of result, sensitivity, specificity and cost. The development of high-throughput methods for typing bacterial pathogens requires careful assessment of the qualities of candidate systems. Therefore, it is extremely important not only to compare methods with each other, but also to define the robustness or weakness of individual methods [45,60,61]. The use of molecular methods for epidemiological typing of nosocomial bacterial pathogens has become a standard service for hospital infection control programs. It is also an important tool for the surveillance of antibiotic-resistant pathogens, such as MRSA strains causing epidemics in health care facilities or other unexpected staphylococci, as a multiresistant Staphylococcus hominis infecting a dyalisis patient (Macía M., Méndez-Alvarez S. et al., 2012, unpublished results).

\section{Acknowledgements}

This work was partially supported by grants FIS06/0002 and FIS10/00125 from INSTITUTO DE SALUD CARLOSIII (Spanish Health Ministry) to S.M.-A.

\section{REFERENCES}

[1] E. M. C. Nright, D. A. Robinson, G. Randle, E. J. Feil, H. Grundmann and B. G. Spratt, "The Evolutionary History of Methicillin-Resistant Staphylococcus aureus (MRSA)," Proceedings of the National Academy of Sciences, Vol. 99, No. 11, 2002, pp. 7687-7692. doi:10.1073/pnas.122108599

[2] A. S. Field, J. Y. Paik, D. Stark, M. R. Qiu, A. Morey, M.
L. Plit, E. U. Canning and A. R. Glanville, "Myositis Due to the Microsporidian Anncaliia (Brachiola) Algerae in a Lung Transplant Recipient," Transplant Infectious Disease, Vol. 14, No. 2, 2012, pp. 169-176. doi:10.1111/j.1399-3062.2012.00724.x

[3] N. E. Hiemann, S. Grimmer, D. Kemper, C. Knosalla and R. Hetzer, "Tuberculous Meningitis in a Lung Transplanted Patient," Transplant Infectious Disease, Vol. 14, No. 4, 2012, pp. E19-E22. doi:10.1111/j.1399-3062.2012.00736.x

[4] J. G. Mainz, J. Hentschel, C. Schien, N. Cramer, W. Pfister, J. F. Beck and B. Tümmler, "Sinonasal Persistence of Pseudomonas Aeruginosa after Lung Transplantation," Journal of Cystic Fibrosis, Vol. 11, No. , 2012, pp. 158161. doi:10.1016/j.jcf.2011.10.009

[5] S. M. Palmer, A. P. Limaye, M. Banks, D. Gallup, J. Chapman, E. C. Lawrence, J. Dunitz, A. Milstone, J. Reynolds, G. L. Yung, K. M. Chan, R. Aris, E. Garrity, V. Valentine, J. McCall, S. C. Chow, R. D. Davis and R. Avery, "Extended Valganciclovir Prophylaxis to Prevent Cytomegalovirus after Lung Transplantation: A Randomized, Controlled Trial," Annals Internal Medicine, Vol. 152, No. 12, 2010, pp. 761-769.

[6] N. Singh and S. Husain, "Aspergillus Infections after Lung Transplantation: Clinical Differences in Type of Transplant and Implications for Management," The Journal of Heart and Lung Transplantation, Vol. 22, No. 3, 2003, pp. 258-266. doi:10.1016/S1053-2498(02)00477-1

[7] P. Tabarsi, A. Soraghi, M. Marjani, P. Zandian, P. Baghaei, K. Najafizadeh, A. Droudinia, S. A. Sarrafzadeh, P. Javanmard and D. Mansouri, "Comparison of Serum and Bronchoalveolar Lavage Galactomannan in Diagnosing Invasive Aspergillosis in Solid-Organ Transplant Recipients," Experimental Clinical Transplantation, Vol. 10, No. 3, 2012, pp. 278-281. doi:10.6002/ect.2011.0176

[8] E. Lindsay and Nicolle, "Infection Control Programmes to Contain Antimicrobial Resistance," World Health Organization, Geneva, 2001. doi:10.1046/j.1468-3148.2001.00071.x

[9] J. Arranz, A. Soriano, I. Garcia, I. García, M. T. Concepción, J. Navarro, A. Arteaga, X. Filella, P. Bravo, M. Barrera, S. Escribano, A. Jiménez, E. Moneva, J. Fernandez, A. Varona, E. Herrero and D. Dominguez, "Effect of Proinflammatory Cytokines (IL-6, TNF-Alpha, IL-1beta) on Hemodynamic Performance during Orthotopic Liver Transplantation," Transplantation Proceedings, Vol. 35, No. 5, 2003, pp. 1884-1887. doi:10.1016/S0041-1345(03)00603-1

[10] A. T. Braun and C. A. Merlo, "Cystic Fibrosis Lung Transplantation," Current Opinion in Pulmonary Medicine, Vol. 17, No. 6, 2011, pp. 467-472.

[11] D. Dhar, J. L. Dickson, M. R. Carby, H. S. Lyster, A. V. Hall and N. R. Banner, "Fungal Infection in Cardiothoracic Transplant Recipients: Outcome without Systemic Amphotericin Therapy," Transplant International, Vol. 25, No. 7, 2012, pp. 758-764. doi:10.1111/j.1432-2277.2012.01499.x

[12] C. López-Aguilar, E. Perez-Roth, A. Moreno, M. C. Duran, C. Casanova, A. Aguirre-Jaime, S. Mendez- 
Alvarez, "Association between the Presence of the Panton-Valentine Leukocidin-Encoding Gene and a Lower Rate of Survival among Hospitalized Pulmonary Patients with Staphylococcal Disease," Journal of Clinical Microbiology, Vol. 45, No. 1, 2007, pp. 274-276.

doi:10.1128/JCM.02032-06

[13] F. S. Herrero and J. B. Olivas, "Microbiology and Risk Factors for Community-Acquired Pneumonia," Seminars in Respiratory and Critical Care Medicine, Vol. 33, No. 3, 2012, pp. 220-231. doi:10.1055/s-0032-1315634

[14] E. Hodson, J. Craig, G. Strippoli and A. C. Webster, “Antiviral Medications for Preventing Cytomegalovirus Disease in Solid Organ Transplant Recipients," Cochrane Database of Systemic Reviews 16, 2008, Article ID: CD003774.

[15] D. F. Florescu, F. Qiu, R. Vivekanandan, D. F. Mercer, A. N. Langnas and A. C. Kalil, "Risk Factors and Outcomes of Staphylococcus aureus Infections after Small Bowel and Multivisceral Transplantation," Pediatric Infectious Disease Journal, Vol. 31, No. 1, 2012, pp. 25-29. doi:10.1097/INF.0b013e3182310fb6

[16] S. J. Peacock, G. D. I de Silva, A. Justice, C. E. Cowland, C. G. Moore, N. P. Winearls and J. Day, "Comparison of Multilocus Sequence Typing and Pulsed-Field Gel Electrophoresis as Tools for Typing Staphylococcus aureus Isolates in a Microepidemiological Setting," Journal of Clinical Microbiology, Vol. 40, No. , 2002, pp. 37643777. doi:10.1128/JCM.40.10.3764-3770.2002

[17] R. W. Bonvillain, V. G. Valentine, G. Lombard, S. LePlace, G. Dhillon and G. Wang, "Post-Operative Infections in Cystic Fibrosis and Non-Cystic Fibrosis Patients after Lung Transplantation," The Journal of Heart and Lung Transplantation, Vol. 26, No. 9, 2007, pp. 890-897. doi:10.1016/j.healun.2007.07.002

[18] J. D. Christie, L. B. Edwards, P. Aurora, F. Dobbels, R. Kirk, A. O. Rahmel, D. O. Taylor, A. Y. Kucheryavaya and M. I. Hertz, "Registry of the International Society for Heart and Lung Transplantation: Twenty-Fifth Official Adult Lung and Heart/Lung Transplantation Report," Journal of Heart and Lung Transplantation, Vol. 27, No. 9, 2008, pp. 957-969. doi:10.1016/j.healun.2008.07.018

[19] A. Copca-Álvarez, M. A. Cabrera-Suarez, G. PulidoReyes, J. Alcoba-Florez, M. Morales and S. MendezAlvarez, "Coagulase Negative Staphylococcus Causes Catheter Associated Bacteriemia in a Patient with Esophagus Adecarcinome," Journal of Cancer Therapy, Vol. 3, No. 5, 2012, pp. 627-629.

[20] Laboratory of Health Care Associated Infection (LHCAI), "Community Skin Infection Study," Staphylococcus Reference Unit (SRU), Health Protection Agency, Centre for Infections, London.

[21] S. R. Burguete, D. J. Maselli, J. F. Fernandez and S. M. Levine, "Lung Transplant Infection," Respirology, 2012. doi:10.1111/j.1440-1843.2012.02196.x

[22] J. Hafkin and E. Blumberg, "Infections in Lung Transplantation: New Insights," Current Opinion in Organ Transplantation, Vol. 14, No. 5, 2009, pp. 483-487. doi:10.1097/MOT.0b013e32833062f6

[23] K. D. Sims, E. A. Blumberg, "Common Infections in the
Lung Transplant Recipient," Clinics in Chest Medicine, Vol. 32, No. 2, 2011. 327-341. doi:10.1016/j.ccm.2011.02.010

[24] A. Humar and D. Snydman, "Cytomegalovirus in Solid Organ Transplant Recipients," American Journal of Transplantation, Vol. 9, Suppl. 4, 2009, pp. S78-S86. doi:10.1111/j.1600-6143.2009.02897.x

[25] S. Husain, "Unique Characteristics of Fungal Infections in Lung Transplant Recipients," Clinics in Chest Medicine, Vol. 30, No. 2, 2009, pp. 307-313. doi:10.1016/j.ccm.2009.03.002

[26] M. L. Green, W. Leisenring, D. Stachel, S. A. Pergam, B. M. Sandmaier, A. Wald, L. Corey and M. Boeckh, "Efficacy of a Viral Load-Based, Risk-Adapted, Preemptive Treatment Strategy for Prevention of Cytomegalovirus Disease after Hematopoietic Cell Transplant," Biology of Blood and Marrow Transplantation, 2012. doi:10.1016/j.bbmt.2012.05.015

[27] M. Zancolli, P. Canepa, A. Ceravolo, V. Parodi, F. Ansaldi, "Determinants of Invasiveness and Ability to Cause Invasive Pneumococcal Disease, Pneumonia and Acute Otitis Media of Different Serotypes of Streptococcus pneumoniae," Journal of Preventive Medicine and Hygiene, Vol. 52, No. 2, 2011, pp. 47-54.

[28] C. Benden, P. Aurora, L. B. Edwards, A. Y. Kucheryavaya, J. D. Christie, F. Dobbels, R. Kirk, A. O. Rahmel, J. Stehlik and M. I. Hertz, "The Registry of the International Society for Heart and Lung Transplantation: Fourteenth Pediatric Lung and Heart-Lung Transplantation Report 2011," The Journal of Heart and Lung Transplantation, Vol. 30, No. 10, 2011, pp. 1123-1132. doi:10.1016/j.healun.2011.08.011

[29] F. P. Silveira and S. Husain, "Fungal Infections in Lung Transplant Recipients," Current Opinion Pulmonary Medicaine, Vol. 14, No. 3, 2008, pp. 211-218. doi:10.1097/MCP.0b013e3282f9b1d1

[30] A. M. Rivera and H. W. Boucher, "Current Concepts in Antimicrobial Therapy against Select Gram-Positive Organisms: Methicillin-Resistant Staphylococcus aureus, Penicillin-Resistant Pneumococci, and Vancomycin-Resistant Enterococci," Mayo Clinic Proceedings, Vol. 86, No. 12, 2011, pp. 1230-1243. doi: $10.4065 / \mathrm{mcp} .2011 .0514$

[31] V. B. Kute, M. P. Patel, S. B. Patil, P. R. Shah, A. V. Vanikar, M. R. Gumber, H. V Patel, J. D. Shrimali and H. L. Trivedi, "Bronchiolitis Obliterans Organizing Pneumonia (BOOP) after Renal Transplantation," International Urology and Nephrology, 2012. doi:10.1007/s11255-012-0182-4

[32] E. Pérez-Roth, J. Alcoba-Flórez, C. López-Aguilar, B. Rivero-Pérez, I. Gutiérrez-González and S. MéndezÁlvarez, "A Case of Familial Furunculosis Associated to Community-Acquired Leukocidin-Positive MethicillinSusceptible Staphylococcus aureus ST152," Journal of Clinical Microbiology, Vol. 48, No. 1, 2010, pp. 329-332. doi:10.1128/JCM.00622-09

[33] J. Štěpán, R. Pantůček and J. Doškař, "Molecular Diagnostics of Clinically Important Staphylococci," Folia Mi crobiologica, Vol. 49, No. 4, 2004, pp. 353-386. 
[34] A. Sole and M. Salavert, "Fungal Infections after Lung Transplantation," Current Opinion Pulmonary Medicaine, Vol. 15, No. 3, 2009, pp. 243-253. doi:10.1097/MCP.0b013e328326f410

[35] S. Méndez-Alvarez and E. Pérez-Roth, "Multiplex PCR in Clinical Microbiology," Enfermedades Infecciosas y Microbiologia Clinica, Vol. 22, No. 3, 2004, pp. 183-191. doi:10.1157/13058027

[36] E. Pérez-Roth, F. Lorenzo-Díaz, N. Batista, A. Moreno and S. Méndez-Álvarez, "Tracking Methicillin-Resistant Staphylococcus aureus Clones during a 5-Year Period (1998 to 2002) in a Spanish Hospital," Journal of Clinical Microbiology, Vol. 42, No. 10, 2004, pp. 4649-4656. doi:10.1128/JCM.42.10.4649-4656.2004

[37] E. Pérez-Roth, F. Claverie-Martín, J. Villar and S. Méndez-Alvarez, "Multiplex PCR for Simultaneous Identification of Staphylococcus aureus and Detection of Methicillin and Mupirocin Resistance," Journal of Clinical Microbiology, Vol. 39, No. 11, 2001, pp. 4037-4041. doi:10.1128/JCM.39.11.4037-4041.2001

[38] E. Ramos-Trujillo, E. Pérez-Roth, S. Méndez-Alvarez and F. Claverie-Martín, "Multiplex PCR for Simultaneous Detection of Enterococcal Genes vanA and vanB and Staphylococcal Genes mecA, ileS-2 and femB," International Microbiology, Vol. 6, No. 2, 2003, pp. 113-115. doi:10.1007/s10123-003-0118-Z

[39] J. Donate-Correa, J. Alcoba-Flórez and S. Méndez- Álvarez, "New Staphylococcus aureus Genetic Cluster Associated with Infectious Osteomyelitis," International Microbiology, Vol. 14, No. 1, 2011, pp. 33-39.

[40] E. Pérez-Roth, S. M Kwong, J. Alcoba-Florez, N. Firth and S. Méndez-Alvarez, "Complete Nucleotide Sequence and Comparative Analysis of pPR9, a 41.7-Kilobase Conjugative Staphylococcal Multiresistance Plasmid Conferring High-Level Mupirocin Resistance," Antimicrobial Agents and Chemotherapy, Vol. 54, No. 5, 2010, pp. 2252-2257. doi:10.1128/AAC.01074-09

[41] E. Pérez-Roth, C.López-Aguilar, J. Alcoba-Florez and S. Méndez-Alvarez, "High-Level Mupirocin Resistance within Methicillin-Resistant Staphylococcus aureus Pandemic Lineages," Antimicrobial Agents and Chemotherapy, Vol. 50, No. 9, 2006, pp. 3207-3211. doi:10.1128/AAC.00059-06

[42] E. Pérez-Roth, E. Armas-González, J. Alcoba-Flórez and S. Méndez-Álvarez, "PCR-Based Amplification of Heterogeneous IS257-ileS2 Junctions for Molecular Monitoring of High-Level Mupirocin Resistance in Staphylococci," Journal of Antimicrobial Chemotherapy, Vol. 66, No. 3, 2011. doi:10.1093/jac/dkq493

[43] E. Pérez-Roth, F. Lorenzo-Díaz and S. Méndez-Alvarez, "Establishment and Clonal Dissemination of the Methicillin-Resistant Staphylococcus aureus UK-16 Epidemic Strain in a Spanish Hospital," Journal of Clinical Microbiology, Vol. 41, No. 11, 2003, p. 5353. doi:10.1128/JCM.41.11.5353.2003

[44] B. Rivero-Pérez, J. Alcoba-Florez and S. Méndez-Alvarez, "Evaluation of Multiple-Locus Variable-Number Tandem-Repeat Analysis for Typing Polyclonal Hospital- and Community-Acquired Methicillin-Resistant Staphylococ- cus aureus Populations," Concept Press Ltd., Long Island City, 2012 (in press).

[45] F. C. Tenover, , R. R. Vaughn, L. K. McDougal, G. E. Fosheim and J. E. McGowan Jr., "Multiple-Locus Variable-Number Tandem-Repeat Assay Analysis of Methicillin-Resistant Staphylococcus aureus Strains," Journal of Clinical Microbiology, Vol. 45, No. 7, 2007, PP. 2215-2219. doi:10.1128/JCM.02451-06

[46] T. Ueno, S. Toyooka, J. Soh, K. Miyoshi, S. Sugimoto, M. Yamane, T. Oto and S. Miyoshi, "Intrathoracic Irrigation with Arbekacin for Methicillin-Resistant Staphylococcus aureus Empyema Following Lung Resection," Interactive Cardiovascular and Thoracic Surgery, Vol. 15, No. 3, 2012. doi:10.1093/icvts/ivs285

[47] G. D. de Silva, A. Justice, A. R. Wilkinson, J. Buttery, M. Herbert, N. P. Day and S. J. Peacock, "Genetic Population Structure of Coagulase-Negative Staphylococci Associated with Carriage and Disease in Preterm Infants," Clinical Infectious Diseases, Vol. 33, No. 9, 2001, pp. 1520-1528. doi:10.1086/323338

[48] M. E. Kaufmann, "Pulse-Field Gel Electrophoresis," In: N. Woodford and A. P. Johnson, Eds., Molecular Bacteriology. Protocols and Clinical Applications, Humana Press, Totowa, 1998, pp. 33-50. doi:10.1385/0-89603-498-4:33

[49] A. Singh, R. V. Goering, S. Simjee, S. L. Foley and M. J. Zervos, "Application of Molecular Techniques to the Study of Hospital Infection," Clinical Microbiology Reviews, Vol. 19, No. 3, 2006, 512-530. doi:10.1128/CMR.00025-05

[50] H. Al-Talib, C. Y. Yean, A. Al-Khateeb, H. Hassan, K.-K. B. Singh, K. Al-Jashamy and M. Ravichandran, "A Pentaplex PCR Assay for the Rapid Detection of Methicillin-Resistant Staphylococcus aureus and Panton-Valentine Leucocidin," BMC Microbiology, Vol. 9, 2009, p. 113. doi:10.1186/1471-2180-9-113

[51] A. Sabat, , J. Krzyszton-Russjan, W. Strzalka, R. Filipek, K. Kosowska, W. Hryniewicz, J. Travis and J. Potempa, "New Method for Typing Staphylococcus aureus Strains: Multiple-Locus Variable-Number Tandem-Repeat Analysis of Polymorphism and Genetic Relationships of Clinical Isolates," Journal of Clinical Microbiology, Vol. 41, No. 4, 2003, pp. 1801-1804. doi:10.1128/JCM.41.4.1801-1804.2003

[52] B. Rivero-Pérez, E. Pérez-Roth and S. Méndez-Alvarez, "Evaluation of Multiple-Locus Variable-Number Tandem-Repeat Analysis for Typing a Polyclonal Hospital-Acquired Methicillin-Resistant Staphylococcus aureus Population in an Area Where Such Infections Are Endemic," Journal of Clinical Microbiology, Vol. 48, No. 8, 2010, pp. 2991-2994. doi:10.1128/JCM.02220-09

[53] B. Rivero-Pérez, J. Alcoba-Flórez and S. Méndez-Álvarez, "Genetic Diversity of Community-Associated Methicillin-Resistant Staphylococcus aureus Isolated from Tenerife Island, Spain," Infection, Genetics and Evolution, Vol. 12, No. 3, 2012, pp. 586-590. doi:10.1016/j.meegid.2012.02.007

[54] M. A. Abramson and D. J. Sexton, "Nosocomial Methicillin-Resistant and Methicillin-Susceptible Staphylococ- 
cus aureus Primary Bacteremia: At What Costs?" Infection Control and Hospital Epidemiology, Vol. 20, No. 6, 1999, pp. 408-411. doi:10.1086/501641

[55] A. J. Sabat, M. A. Chlebowicz, H. Grundmann, J. P. Arends, G. Kampinga, N. E. Meessen, A. W. Friedrich, J. M. van Dijl, "Microfluidic-Chip-Based Multiple-Locus Variable-Number Tandem-Repeat Fingerprinting with New Primer Sets for Methicillin-Resistant Staphylococcus aureus," Journal of Clinical Microbiology, Vol. 50, No. 7, 2012, pp. 2255-2262. doi:10.1128/JCM.00056-12

[56] D. Harmsen, H. Claus, W. Witte, J. Rothganger, H. Claus, D. Turnwald and U. Vogel, "Typing of Methicillin-Resistant Staphylococcus aureus in a University Hospital Setting by Using Novel Software for spa Repeat Determination and Database Management," Journal of Clinical Microbiology, Vol. 41, No. 12, 2003, pp. 54425448. doi:10.1128/JCM.41.12.5442-5448.2003

[57] M. C. Enright, N. P. Day, C. E. Davies, S. J. Peacock and B. G. Spratt, "Multilocus Sequence Typing for Characterization of Methicillin-Resistant and Methicillin Susceptible Clones of Staphylococcus aureus," Journal of Clinical Microbiology, Vol. 38, No. 3, 2000, pp. 10081015.

[58] E. J. Feil, J. E. Cooper, H. Grundmann, D. A. Robinson, M. C. Enright, T. Berendt, S. J. Peacock, J. M. Smith, M.
Murphy, B. G. Spratt, C. E. Moore and N. P. Day, "How Clonal Is Staphylococcus aureus?" Journal of Bacteriology, Vol. 185, No. 11, 2003, pp. 3307-3316. doi:10.1128/JB.185.11.3307-3316.2003

[59] D. C. Oliveira and H. de Lencastre, "Multiplex PCR Strategy for Rapid Identification of Structural Types and Variants of the mec Element in Methicillin-Resistant Staphylococcus aureus," Antimicrobial Agents and Chemotherapy, Vol. 46, No. 7, 2002, pp. 2155-2161. doi:10.1128/AAC.46.7.2155-2161.2002

[60] F. C. Tenover, R. D. Arbeit and R. V. Goering, "How to Select and Interpret Molecular Strain Typing Methods for Epidemiological Studies of Bacterial Infections: A Review for Healthcare Epidemiologists. Molecular Typing Working Group of the Society for Healthcare Epidemiology of America," Infection Control and Hospital Epidemiology, Vol. 18, No. 6, 1997, pp. 426-439. doi:10.1086/647644

[61] F. C. Tenover, R. D. Arbeit, R. V. Goering, P. A. Mickelsen, B. E. Murray, D. H. Persing and B. Swaminathan, "Interpreting Chromosomal DNA Restriction Patterns Produced by Pulsed-Field Gel Electrophoresis: Criteria for Bacterial Strain Typing," Journal of Clinical Microbiology, Vol. 33, No. 9, 1995, pp. 2233-2239. 